\title{
Numerical solutions of the Kawahara equation by the septic B-spline collocation method
}

\author{
Battal Gazi Karakoç ${ }^{1, *}$, Halil Zeybek ${ }^{2}$ and Turgut $\mathrm{Ak}^{3}$ \\ ${ }^{1}$ Nevsehir Haci Bektas Veli University, Nevsehir, 50300, Turkey \\ ${ }^{2}$ Abdullah Gul University, Kayseri, 38039, Turkey \\ ${ }^{3}$ Armutlu Vocational High School, Yalova University, 77500, Yalova, Turkey
}

Received 4 April 2014; Accepted 17 May 2014

Editor: David G. Yu

\begin{abstract}
In this article, a numerical solution of the Kawahara equation is presented by septic B-spline collocation method. Applying the Von-Neumann stability analysis, the present method is shown to be unconditionally stable. The accuracy of the proposed method is checked by two test problems. $L_{2}$ and $L_{\infty}$ error norms and conserved quantities are given at selected times. The obtained results are found in good agreement with the some recent results.
\end{abstract}

Keywords Kawahara equation, Finite element method, Septic B-Splines, Collocation, Solitary waves.

AMS classification: 74S05, 41A15, 65N35, 76B25.

DOI: $10.19139 /$ soic.v2i3.74

\section{Introduction}

In this paper, we consider the Kawahara equation which is firstly studied by Kawahara[1],

$$
U_{t}+\left(\frac{105}{16}\right) \alpha^{2} U U_{x}+\left(\frac{13}{4}\right) \beta U_{x x x}-U_{x x x x x}=0,
$$

${ }^{*}$ Correspondence to: Nevsehir Haci Bektas Veli University, Nevsehir, 50300, Turkey. E-mail: sbgk44@gmail.com.

ISSN 2310-5070 (online) ISSN 2311-004X (print)

Copyright (c) 2014 International Academic Press 
where $\alpha, \beta$ are nonzero arbitrary constants. This equation is also known as a fifthorder $\mathrm{KdV}$ equation or singularly perturbed $\mathrm{KdV}$ equation[2]. The fifth-order $\mathrm{KdV}$ equation is one of the most known nonlinear evolution equation which is used in the theory of magneto-acoustic waves in a plasma, capillary-gravity waves and in the theory of shallow water waves having surface tension. If the coefficient of the term having third-order derivative is dominant over that of the fifth-order, a monotone solitary wave solution is found. But if the fifth-order derivative is dominating over the third one, oscillatory structure of the solitary waves forms which are called as Kawahara solitons[3]. If we take $\alpha=4 / \sqrt{105}$ and $\beta=4 / 13$, Eq.(1) returns to another form of the equation

$$
U_{t}+U U_{x}+U_{x x x}-U_{x x x x x}=0 .
$$

Various analytical and numerical studies including; Crank-Nicolson Differential quadrature method[3], RBF collocation method[4], meshless method of lines[5], Dual-Petrov Galerkin method[6], Adomian decomposition method[7], tanhfunction method[8], variational iteration and homotopy perturbation method[9] and sine-cosine method[10] have been proposed for solving the Kawahara type equations.

In the present paper, a numerical scheme based on the septic B- spline collocation method has been set up for solving the Kawahara equation with a variant of initial and boundary conditions. This paper is organized as follows: In Section 2, numerical algorithm is presented. In Section 3, stability analysis of the scheme is given and numerical results of the equation are obtained in Section 4. Finally a summary is presented in Section 5.

\section{Septic B-spline finite element solution}

Consider the Kawahara Eq.(2) with the following boundary and initial conditions

$$
\begin{gathered}
U(a, t)=\alpha_{1}, \quad U(b, t)=\alpha_{2}, \quad a \leq x \leq b, \quad t>0, \\
U(x, 0)=f(x) .
\end{gathered}
$$

To implement the numerical algorithm, solution region of the problem is restricted over an interval $a \leq x \leq b$. And let the partition of the space interval $[a, b]$ into uniformly sized finite elements of length $h$ by the knots $x_{m}$ such that $a=x_{0}<x_{1}<\ldots<x_{N}=b$ and $h=\frac{b-a}{N}$. The set of septic B-spline functions $\left\{\phi_{-3}(x), \phi_{-2}(x), \ldots, \phi_{N+2}(x), \phi_{N+3}(x)\right\}$ forms a basis over the problem domain of solution $[a, b]$. A global approximation $U_{N}(x, t)$ is expressed in terms of the 
septic B-splines as

$$
U_{N}(x, t)=\sum_{i=-3}^{N+3} \phi_{i}(x) \delta_{i}(t)
$$

where $\delta_{i}(t)$ are time dependent parameters to be determined from the boundary and collocation conditions. Septic B-splines $\phi_{m}(x),(m=-3,-2, \ldots, N+2, N+$ $3)$ at the knots $x_{m}$ are defined over the interval $[a, b]$ by[11]

$$
\phi_{m}(x)=\frac{1}{h^{7}} \begin{cases}\left(x-x_{m-4}\right)^{7} & {\left[x_{m-4}, x_{m-3}\right]} \\ \left(x-x_{m-4}\right)^{7}-8\left(x-x_{m-3}\right)^{7} & {\left[x_{m-3}, x_{m-2}\right]} \\ \left(x-x_{m-4}\right)^{7}-8\left(x-x_{m-3}\right)^{7}+28\left(x-x_{m-2}\right)^{7} & {\left[x_{m-2}, x_{m-1}\right]} \\ \left(x-x_{m-4}\right)^{7}-8\left(x-x_{m-3}\right)^{7}+28\left(x-x_{m-2}\right)^{7}-56\left(x-x_{m-1}\right)^{7} & {\left[x_{m-1}, x_{m}\right]} \\ \left(x_{m+4}-x\right)^{7}-8\left(x_{m+3}-x\right)^{7}+28\left(x_{m+2}-x\right)^{7}-56\left(x_{m+1}-x\right)^{7} & {\left[x_{m}, x_{m+1}\right]} \\ \left(x_{m+4}-x\right)^{7}-8\left(x_{m+3}-x\right)^{7}+28\left(x_{m+2}-x\right)^{7} & {\left[x_{m+1}, x_{m+2}\right]} \\ \left(x_{m+4}-x\right)^{7}-8\left(x_{m+3}-x\right)^{7} & {\left[x_{m+2}, x_{m+3}\right]} \\ \left(x_{m+4}-x\right)^{7} & {\left[x_{m+3}, x_{m+4}\right]} \\ 0 & \text { otherwise. }\end{cases}
$$

Substituting trial function (6) into Eq.(5), the nodal values of $U, U^{\prime}, U^{\prime \prime}, U^{\prime \prime \prime}, U^{\imath v}, U^{v}$ at the knots $x_{m}$ are obtained in terms of the element parameters $\delta_{m}$ by

$$
\begin{aligned}
& U_{N}\left(x_{m}, t\right)=U_{m}=\delta_{m-3}+120 \delta_{m-2}+1191 \delta_{m-1}+2416 \delta_{m}+1191 \delta_{m+1}+ \\
& 120 \delta_{m+2}+\delta_{m+3}, \quad m=0, \ldots, N \\
& U_{m}^{\prime}=\frac{7}{h}\left(-\delta_{m-3}-56 \delta_{m-2}-245 \delta_{m-1}+245 \delta_{m+1}+56 \delta_{m+2}+\delta_{m+3}\right), \\
& U_{m}^{\prime \prime}=\frac{42}{h^{2}}\left(\delta_{m-3}+24 \delta_{m-2}+15 \delta_{m-1}-80 \delta_{m}+15 \delta_{m+1}+24 \delta_{m+2}+\delta_{m+3}\right) \\
& U_{m}^{\prime \prime \prime}=\frac{210}{h^{3}}\left(-\delta_{m-3}-8 \delta_{m-2}+19 \delta_{m-1}-19 \delta_{m+1}+8 \delta_{m+2}+\delta_{m+3}\right), \\
& U_{m}^{\prime v}=\frac{840}{h^{4}}\left(\delta_{m-3}-9 \delta_{m-1}+16 \delta_{m}-9 \delta_{m+1}+\delta_{m+3}\right) \\
& U_{m}^{v}=\frac{2520}{h^{5}}\left(-\delta_{m-3}+4 \delta_{m-2}-5 \delta_{m-1}+5 \delta_{m+1}-4 \delta_{m+2}+\delta_{m+3}\right) .
\end{aligned}
$$

The B-splines $\phi_{m}(x)$ and its six principle derivatives vanish outside the interval $\left[x_{m-4}, x_{m+4}\right]$.

Now we identify the collocation points with the knots and use Eq.(7) to evaluate $U_{m}$, its necessary space derivatives and substitute into Eq.(2) to obtain the set of the coupled ordinary differential equations:

$$
\begin{aligned}
& \dot{\delta}_{m-3}+120 \dot{\delta}_{m-2}+1191 \dot{\delta}_{m-1}+2416 \dot{\delta}_{m}+1191 \dot{\delta}_{m+1}+120 \dot{\delta}_{m+2}+ \\
& \frac{7 Z_{m}}{h}\left(-\delta_{m-3}-56 \delta_{m-2}-245 \delta_{m-1}+245 \delta_{m+1}+56 \delta_{m+2}+\delta_{m+3}\right)+ \\
& \frac{210}{h^{3}}\left(-\delta_{m-3}-8 \delta_{m-2}+19 \delta_{m-1}-19 \delta_{m+1}+8 \delta_{m+2}+\delta_{m+3}\right)- \\
& \frac{2520}{h^{5}}\left(-\delta_{m-3}+4 \delta_{m-2}-5 \delta_{m-1}+5 \delta_{m+1}-4 \delta_{m+2}+\delta_{m+3}\right)=0
\end{aligned}
$$

where

$$
\begin{aligned}
& Z_{m}=U_{m}= \\
& \delta_{m-3}+120 \delta_{m-2}+1191 \delta_{m-1}+2416 \delta_{m}+1191 \delta_{m+1}+120 \delta_{m+2}+\delta_{m+3},
\end{aligned}
$$


and denotes derivative with respect to time. If time parameters $\delta_{i}$ 's and its time derivatives $\dot{\delta}_{i}$ 's in Eq.(8) are discretized by the Crank-Nicolson formula and usual finite difference aproximation, respectively,

$$
\delta_{i}=\frac{1}{2}\left(\delta^{n}+\delta^{n+1}\right), \quad \dot{\delta}_{i}=\frac{\delta^{n+1}-\delta^{n}}{\Delta t}
$$

we obtain a recurrence relationship between two time levels $n$ and $n+1$ relating two unknown parameters $\delta_{i}^{n+1}, \delta_{i}^{n}$ for $i=m-2, m-1, \ldots, m+1, m+2$

$$
\begin{aligned}
& \gamma_{1} \delta_{m-3}^{n+1}+\gamma_{2} \delta_{m-2}^{n+1}+\gamma_{3} \delta_{m-1}^{n+1}+\gamma_{4} \delta_{m}^{n+1}+\gamma_{5} \delta_{m+1}^{n+1}+\gamma_{6} \delta_{m+2}^{n+1}+\gamma_{7} \delta_{m+3}^{n+1} \quad \text { (10) } \\
= & \gamma_{8} \delta_{m-3}^{n+1}+\gamma_{9} \delta_{m-2}^{n+1}+\gamma_{10} \delta_{m-1}^{n+1}+\gamma_{11} \delta_{m}^{n+1}+\gamma_{12} \delta_{m+1}^{n+1}+\gamma_{13} \delta_{m+2}^{n+1}+\gamma_{14} \delta_{m+3}^{n+1},
\end{aligned}
$$

where

$$
\begin{aligned}
& \gamma_{1}=\left(1-E Z_{m}-M+K\right), \\
& \gamma_{3}=\left(1191-245 E Z_{m}+19 M+5 K\right), \\
& \gamma_{5}=\left(1191+245 E Z_{m}-19 M-5 K\right) \text {, } \\
& \gamma_{7}=\left(1+E Z_{m}+M-K\right) \text {, } \\
& \gamma_{2}=\left(120-56 E Z_{m}-8 M-4 K\right), \\
& \gamma_{4}=(2416) \text {, } \\
& \gamma_{6}=\left(120+56 E Z_{m}+8 M+4 K\right) \text {, } \\
& \gamma_{9}=\left(120+56 E Z_{m}+8 M+4 K\right) \text {, } \\
& \gamma_{11}=(2416) \text {, } \\
& \gamma_{13}=\left(120-56 E Z_{m}-8 M-4 K\right) \text {, } \\
& =\left(1+E Z_{m}+M-K\right) \\
& \gamma_{10}=\left(1191+245 E Z_{m}-19 M-5 K\right) \text {, } \\
& \gamma_{12}=\left(1191-245 E Z_{m}+19 M+5 K\right) \text {, } \\
& \gamma_{14}=\left(1-E Z_{m}-M+K\right) \\
& m=0,1, \ldots, N, \quad E=\frac{7}{2 h} \Delta t, \quad M=\frac{105}{h^{3}} \Delta t, \quad K=\frac{1260}{h^{5}} \Delta t .
\end{aligned}
$$

The system (10) consists of $(N+1)$ linear equations including $(N+7)$ unknown parameters $\left(\delta_{-3}, \delta_{-2}, \delta_{-1} \ldots, \delta_{N+1}, \delta_{N+2}, \delta_{N+3}\right)^{T}$. To obtain a unique solution to this system, we need six additional constraints. These are obtained from the boundary conditions and can be used to eliminate $\delta_{-3}, \delta_{-2}, \delta_{-1}$ and $\delta_{N+1}, \delta_{N+2}, \delta_{N+3}$ from the system (10) which then becomes a matrix equation for the $N+1$ unknowns $d^{n}=\left(\delta_{0}, \delta_{1}, \ldots, \delta_{N}\right)^{T}$ of the form

$$
A \mathbf{d}^{\mathbf{n}+\mathbf{1}}=B \mathbf{d}^{\mathbf{n}} \text {. }
$$

The matrices $A$ and $B$ are $(N+1) \times(N+1)$ septa-diagonal matrices given by

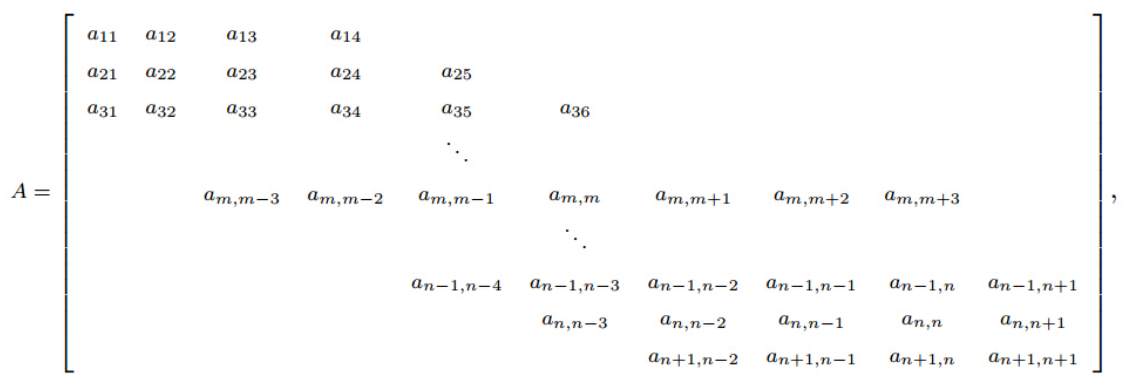

and, for $m=4,5, \ldots n-2$, 


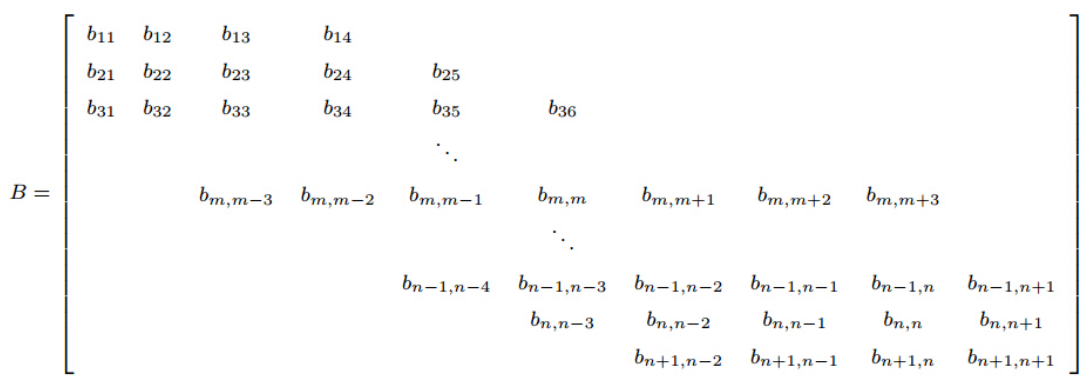

$$
\begin{aligned}
& a_{11}=\frac{186032}{297} E Z_{m}-\frac{108720}{297} K, \\
& a_{12}=\frac{183414}{297} E Z_{m}-\frac{107190}{297} K, \\
& a_{13}=\frac{18480}{297} E Z_{m}-\frac{10800}{297} K, \\
& a_{14}=\frac{154}{297} E Z_{m}-\frac{90}{297} K, \\
& a_{21}=\frac{215411}{297}-\frac{45585}{297} E Z_{m}+\frac{8663}{297} M+\frac{12961}{297} K, \\
& a_{22}=\frac{616822.5}{297}+\frac{18481.5}{297} E Z_{m}+\frac{601.5}{297} M+\frac{10126.5}{297} K, \\
& a_{23}=\frac{340284}{297}+\frac{75168}{297} E Z_{m}-\frac{5640}{297} M-\frac{48}{297} K, \\
& a_{24}=\frac{35525.5}{297}+\frac{8338.5}{297} E Z_{m}+\frac{2378.5}{297} M-\frac{1197.5}{297} K, \\
& a_{25}=1+E Z_{m}+M-K, \\
& a_{31}=\frac{34432}{297}-\frac{7108}{297} E Z_{m}-\frac{1168}{297} M-\frac{2396}{297} K, \\
& a_{32}=\frac{352833}{297}-\frac{71871}{297} E Z_{m}+\frac{6537}{297} M+\frac{591}{297} K, \\
& a_{33}=\frac{717432}{297}+\frac{120}{297} E Z_{m}+\frac{120}{297} M-\frac{120}{297} K, \\
& a_{34}=\frac{353726}{297}+\frac{72766}{297} E Z_{m}-\frac{5642}{297} M-\frac{1486}{297} K, \\
& a_{35}=120+28 E Z_{m}+8 M+4 K, \\
& a_{36}=1+E Z_{m}+M-K,
\end{aligned}
$$

$$
\begin{aligned}
& b_{11}=-\frac{186032}{297} E Z_{m}+\frac{108720}{297} K, \\
& b_{12}=-\frac{183414}{297} E Z_{m}+\frac{107190}{297} K, \\
& b_{13}=-\frac{18480}{297} E Z_{m}+\frac{10800}{297} K, \\
& b_{14}=-\frac{154}{297} E Z_{m}+\frac{90}{297} K, \\
& b_{21}=\frac{215411}{297}+\frac{45585}{297} E Z_{m}-\frac{8663}{297} M-\frac{12961}{297} K, \\
& b_{22}=\frac{616822.5}{297}-\frac{18481.5}{297} E Z_{m}-\frac{601.5}{297} M-\frac{10126.5}{297} K, \\
& b_{23}=\frac{340284}{297}-\frac{75168}{297} E Z_{m}+\frac{5640}{297} M+\frac{48}{297} K, \\
& b_{24}=\frac{35525.5}{297}-\frac{8338.5}{297} E Z_{m}-\frac{2378.5}{297} M+\frac{1197.5}{297} K, \\
& b_{25}=1-E Z_{m}-M+K, \\
& b_{31}=\frac{34432}{297}+\frac{7108}{297} E Z_{m}+\frac{1168}{297} M+\frac{2396}{297} K, \\
& b_{32}=\frac{352833}{297}+\frac{71871}{297} E Z_{m}-\frac{6537}{297} M-\frac{591}{297} K, \\
& b_{33}=\frac{717432}{297}-\frac{120}{297} E Z_{m}-\frac{120}{297} M+\frac{120}{297} K, \\
& b_{34}=\frac{353726}{297}-\frac{72766}{297} E Z_{m}+\frac{5642}{297} M+\frac{1486}{297} K, \\
& b_{35}=120-28 E Z_{m}-8 M-4 K, \\
& b_{36}=1-E Z_{m}-M+K,
\end{aligned}
$$

$$
\begin{array}{rlrl}
a_{m, m-3} & =1-E Z_{m}-M+K, & b_{m, m-3}=1+E Z_{m}+M-K, \\
a_{m, m-2} & =120-28 E Z_{m}-8 M-4 K, & b_{m, m-2}=120+28 E Z_{m}+8 M+4 K, \\
a_{m, m-1}=1191-245 E Z_{m}+19 M+5 K, & b_{m, m-1}=1191+245 E Z_{m}-19 M-5 K, \\
a_{m, m}=2416, & b_{m, m}=2416, \\
a_{m, m+1}=1191+245 E Z_{m}-19 M-5 K, & b_{m, m+1}=1191-245 E Z_{m}+19 M+5 K, \\
a_{m, m+2}=120+28 E Z_{m}+8 M+4 K, & b_{m, m+2}=120-28 E Z_{m}-8 M-4 K, \\
a_{m, m+3}=1+E Z_{m}+M-K, & b_{m, m+3}=1-E Z_{m}-M+K,
\end{array}
$$

Two or three inner iterations are applied to the term $\delta^{n *}=\delta^{n}+\frac{1}{2}\left(\delta^{n}-\delta^{n-1}\right)$ at each time step to cope with the non-linearity caused by $Z_{m}$. Before the commencement of the 


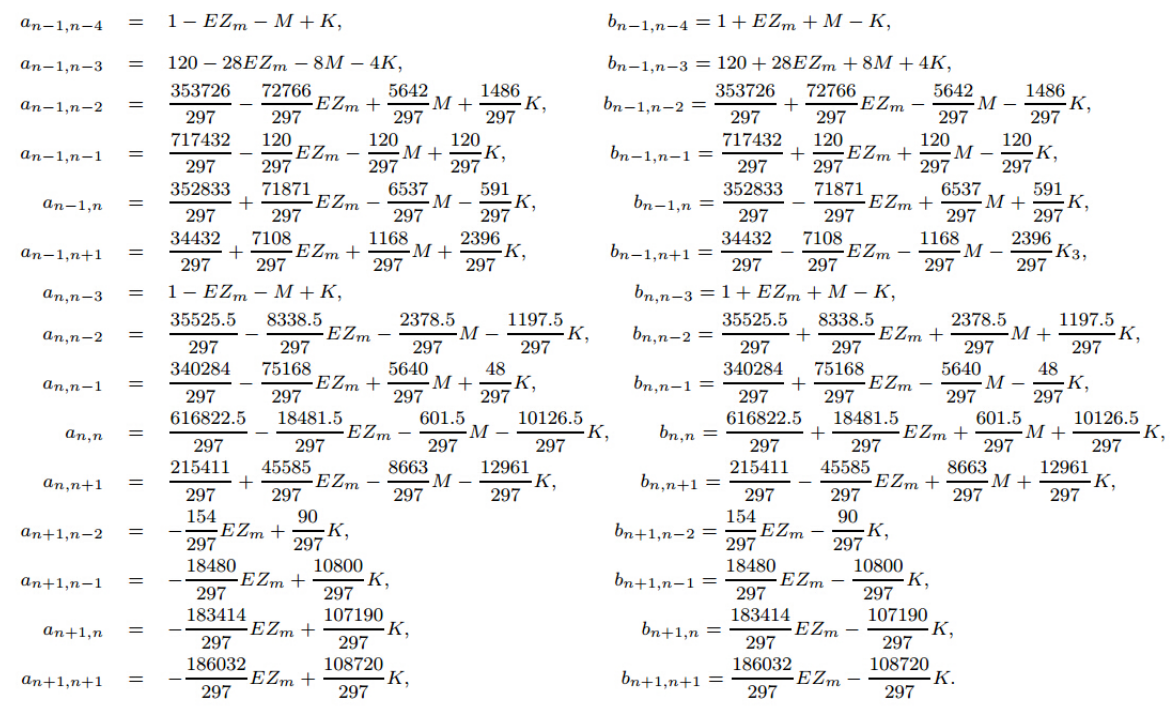

solution process, initial parameters $d^{0}$ must be determined by using the initial condition and following derivatives at the boundaries;

$$
\begin{aligned}
U_{N}(x, 0) & =U\left(x_{m}, 0\right) & & m=0,1,2, \ldots, N \\
\left(U_{N}\right)_{x}(a, 0) & =0, & & \left(U_{N}\right)_{x}(b, 0)=0, \\
\left(U_{N}\right)_{x x}(a, 0) & =0, & & \left(U_{N}\right)_{x x}(b, 0)=0, \\
\left(U_{N}\right)_{x x x}(a, 0) & =0, & & \left(U_{N}\right)_{x x x}(b, 0)=0 .
\end{aligned}
$$

So we have the following matrix form for the initial vector $d^{0}$;

$$
W d^{0}=b,
$$

where

$d^{0}=\left(\delta_{0}, \delta_{1}, \delta_{2}, \ldots, \delta_{N-2}, \delta_{N-1}, \delta_{N}\right)^{T}$

$b=\left(U\left(x_{0}, 0\right), U\left(x_{1}, 0\right), \ldots, U\left(x_{N-1}, 0\right), U\left(x_{N}, 0\right)\right)^{T}$.

\section{A linear stability analysis}

To apply the Von-Neumann stability analysis, the Kawahara equation can be linearized by assuming that the quantity $U$ in the nonlinear term $U U_{x}$ is locally constant. Substituting the Fourier mode $\delta_{j}^{n}=\hat{\delta}^{n} e^{i j k h}(i=\sqrt{-1})$ in which $k$ is a mode number and $h$ is the element size, into the Eq.(10) gives the growth factor $g$ of the form

$$
g=-\frac{a+i b}{a-i b},
$$

Stat., Optim. Inf. Comput. Vol. 2, September 2014. 
$W=\left[\begin{array}{ccccccccccc}1536 & 2712 & 768 & 24 & & & & & & \\ \frac{82731}{81} & \frac{210568.5}{81} & \frac{104796}{81} & \frac{10063.5}{81} & 1 & & & & & \\ \frac{9600}{81} & \frac{96597}{81} & \frac{195768}{81} & \frac{96474}{81} & 120 & 1 & & & & \\ & & \ddots & & & & & & & \\ & & & & & & & & & \\ & & & 1 & 120 & 1191 & 2416 & 1191 & 120 & 1 \\ & & & & 1 & 120 & \frac{96474}{81} & \frac{195768}{81} & \frac{96597}{81} & \frac{9600}{81} \\ & & & & & 1 & \frac{10063.5}{81} & \frac{104796}{81} & \frac{210568.5}{81} & \frac{82731}{81} \\ & & & & & & 24 & 768 & 2712 & 1536\end{array}\right]$

where

$$
\begin{aligned}
a= & \left(19 M+5 T-245 E Z_{m}\right) \sin [h k]+\left(-8 M-4 T-56 E Z_{m}\right) \sin [2 h k]+ \\
& \left(-M+T-E Z_{m}\right) \sin [3 h k], \\
b= & -1208-1191 \cos [h k]-120 \cos [2 h k]-\cos [3 h k] .
\end{aligned}
$$

The modulus of $|g|$ is 1 , therefore the linearized scheme is unconditionally stable.

\section{Numerical examples and results}

To obtain the approximate solutions of the Eq.(2), we consider some numerical experiments including: motion of single solitary wave and interaction of two solitary waves. To show how good the numerical scheme predicts the position and amplitude of the solution as the simulation proceeds, we use the error norm $L_{2}$

$$
L_{2}=\left\|U^{\text {exact }}-U_{N}\right\|_{2} \simeq \sqrt{h \sum_{J=1}^{N}\left|U_{j}^{\text {exact }}-\left(U_{N}\right)_{j}\right|^{2}},
$$

and the error norm $L_{\infty}$

$$
L_{\infty}=\left\|U^{\text {exact }}-U_{N}\right\|_{\infty} \simeq \max _{j}\left|U_{j}^{\text {exact }}-\left(U_{N}\right)_{j}\right|, \quad j=1,2, \ldots, N-1 .
$$

The equation possesses the lowest three conserved quantities given by[12]

$$
\begin{aligned}
& I_{1}=\int_{-\infty}^{\infty} U d x \simeq h \sum_{i=1}^{N} U_{i}^{n}, \\
& I_{2}=\int_{-\infty}^{\infty} \frac{1}{2} U^{2} d x \simeq h \sum_{i=1}^{N} \frac{1}{2}\left(U_{i}^{n}\right)^{2}, \\
& I_{3}=\int_{-\infty}^{\infty}\left(\frac{13}{8} \beta U_{x}^{2}-\frac{105}{96} \alpha^{2} U^{3}+\frac{1}{2} U_{x x}^{2}\right) d x \simeq \\
& h \sum_{i=1}^{N} \frac{13}{8} \beta\left(\left(U_{x}\right)_{i}^{n}\right)^{2}-\frac{105}{96} \alpha^{2}\left(U_{i}^{n}\right)^{3}+\frac{1}{2}\left(\left(U_{x x}\right)_{i}^{n}\right)^{2}
\end{aligned}
$$

We can calculate the integrals (14) by using the trapezium rule and the variables $U_{i}^{n}$ and their derivatives are obtained from Eq.(7). To show the efficient of the numerical 
scheme, the above conserved quantities are expected to remain constant during the run of the algorithm so conserved quantities will be monitored.

\subsection{The motion of single solitary wave}

For this problem, Eq.(2) has a solitary wave solution of the form

$$
U(x, t)=\left(\frac{\beta}{\alpha}\right)^{2} \sec h^{4}\left[\frac{1}{4} \sqrt{\beta}\left(x-\frac{36}{169} t-x_{0}\right)\right]
$$

where $\left(\frac{\beta}{\alpha}\right)^{2}$ is the amplitude of the single solitary wave[13]. It represents a single solitary wave initially centred on $x_{0}$ and moving to the right with velocity $V=(1.5 \beta)^{2}$. Initial condition

$$
U(x, 0)=\left(\frac{\beta}{\alpha}\right)^{2} \sec h^{4}\left[\frac{1}{4} \sqrt{\beta}\left(x-x_{0}\right)\right],
$$

and the boundary conditions $\alpha_{1}=0, \alpha_{2}=0$ are taken at the boundaries.

The values of the parameters $h=0.2, \Delta t=0.001, x_{0}=2, \alpha=4 / \sqrt{105}, \beta=4 / 13$ are chosen over the spatial interval $[-20,30]$ to coincide with that of previous papers[35]. For these parameters, the single solitary wave has an amplitude $A=\left(\frac{\beta}{\alpha}\right)^{2}=0.6213$ and velocity $V=(1.5 \beta)^{2}=0.2130$. Numerical computations are done up to time $t=25$ to obtain the invariants and error norms $L_{2}$ and $L_{\infty}$ at various times. Error norms, three conserved quantities, amplitudes and peak position of the solitary waves are listed in Table $(I)$. It is clearly seen from the table that the invariants remain almost constant during the computer run. Table $(I I)$ shows a comparison of the values of the invariants and error norms obtained by the present method with obtained by the methods[3-5]. It is observed from the table that the error norms obtained by our method is smaller than given in Ref.[3] and almost the same in Refs.[4, 5]. Values of the invariants are also found in good agreement with the others. Solitary wave profiles with $h=0.2, \Delta t=0.001$ are depicted at different time levels in Fig.(1). As it is seen from the figure, the soliton moves to the right at a constant speed and almost unchanged amplitude as time increases, as expected.

Table I. The invariants and the error norms for single solitary wave with $h=0.2, \Delta t=$ $0.001,-20 \leq x \leq 30$.

\begin{tabular}{cccccccc}
\hline$t$ & $I_{1}$ & $I_{2}$ & $I_{3}$ & $L_{2} \times 10^{3}$ & $L_{\infty} \times 10^{3}$ & Amp. & Peak Position \\
\hline 0 & 5.9736088 & 1.2725033 & -0.1645840 & 0.0000 & 0.0000 & 6.2130 & 2 \\
5 & 5.9738529 & 1.2725034 & -0.1620645 & 0.3249 & 0.1116 & 6.2120 & 3 \\
10 & 5.9738517 & 1.2725033 & -0.1633093 & 0.2469 & 0.0852 & 6.2118 & 4.2 \\
15 & 5.9737831 & 1.2725033 & -0.1640871 & 0.1807 & 0.0744 & 6.2130 & 5.2 \\
20 & 5.9737261 & 1.2725033 & -0.1643599 & 0.1529 & 0.0548 & 6.2121 & 6.2 \\
25 & 5.9736181 & 1.2725033 & -0.1644699 & 0.1395 & 0.0511 & 6.2116 & 7.4 \\
\hline
\end{tabular}

\subsection{Interaction of two solitary waves}

In this section, we consider Eq.(2) with the boundary conditions $U(-50)=$ $U(100)=0$, by using the following initial condition 
Table II. The errors and the invariants for single solitary wave with $h=0.2, \Delta t=0.001$, $-20 \leq x \leq 30$, at $t=25$.

\begin{tabular}{lccccc}
\hline Method & $I_{1}$ & $I_{2}$ & $I_{3}$ & $L_{2} \times 10^{3}$ & $L_{\infty} \times 10^{3}$ \\
\hline Present & 5.97361 & 1.27250 & -0.16446 & 0.139 & 0.051 \\
[3](PDQ) & 5.97353 & 1.27250 & -0.16457 & 2.851 & 0.863 \\
[3](CDQ) & 5.97350 & 1.27250 & -0.16458 & 0.159 & 0.076 \\
[4] & 5.97367 & 1.27250 & -0.16459 & 0.093 & 0.023 \\
[5](GA) & 5.97353 & 1.27250 & -0.16458 & 0.131 & 0.039 \\
[5](MQ) & 5.97355 & 1.27250 & -0.16458 & 0.168 & 0.046 \\
\hline
\end{tabular}

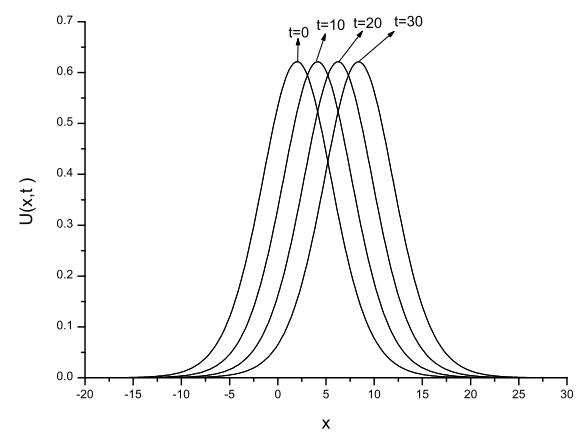

Figure 1. Single solitary wave with $h=0.2, \Delta t=0.001,-20 \leq x \leq 30, t=0,10,20$ and 30 .

$$
U(x, 0)=\sum_{i=1}^{2} A_{i}^{2} \sec h^{4}\left(\frac{\sqrt{\alpha A_{i}}}{4}\left[x-x_{i}\right]\right),
$$

where $\alpha=4 / \sqrt{105}, A_{i}=\beta_{i} / \alpha, \quad \beta_{i}=(12-2 i) / 13, \Delta t=0.01, \quad h=0.2$. Eq.(15) represents two solitary waves having different amplitudes, one with amplitude $A_{1}$ placed initially at $x_{1}=0$ and the second with amplitude $A_{2}$ placed at $x_{2}=20$.

A comparison of the values of the invariants obtained by the present method with those obtained in Refs.[3-5] given in Table $(I I I)$. We notice that the obtained values of the invariants remain almost constant during the computer run. They are also found to be very close with the other earlier papers. Fig.(2) shows the interaction of solitary waves. As it is seen from the figure, at $t=0$, a wave with larger amplitude is on the left of the another wave with smaller amplitude. The larger wave catches up with the smaller one as the time increases. Interaction started at about time $t=20$, overlapping processes occurred between times $t=20$ and $t=40$ and, waves started to resume their original shapes after time $t=40$. At $t=50$, the amplitude of larger waves is 4.36993 at the point $x=83.4$ whereas the amplitude of the smaller one is 2.75247 at the point $x=62.8$. It is found that the absolute difference in amplitude is $2.67 \times 10^{-1}$ for the smaller wave and $4.86 \times 10^{-1}$ for the larger wave. Also, oscillations of small amplitude trailing behind the solitary waves were observed in the Figure. 

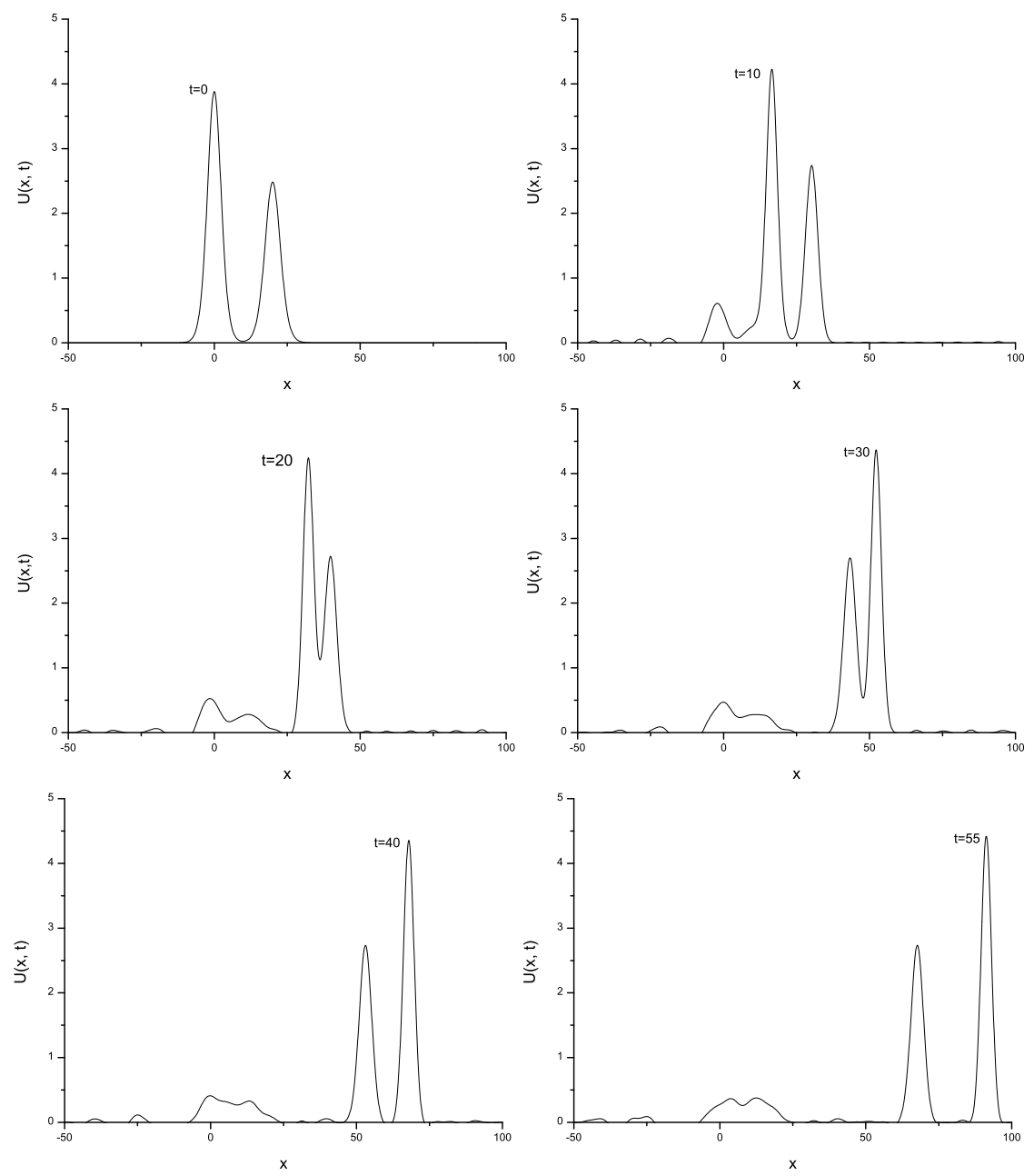

Figure 2. Interaction of two solitary waves with $t=0,10,20,30,40,55$.

\section{Conclusion}

In this paper, a numerical scheme based on the septic B-spline collocation method have been set up to find numerical solution of the Kawahara equation. Unconditional stability is shown by using Von-Neumann analysis. To show the performance of the method, we have examined the motion of a solitary wave and the interaction of two solitary waves. Efficiency and accuracy of the method is shown by calculating $L_{2}, L_{\infty}$ error norms and $I_{1}, I_{2}$ and $I_{3}$ invariant quantities. The obtained results show that the error norms are adequately small and the conservation laws are reasonably well satisfied for 
Table III. Comparison of the invariants for the interaction of two solitary waves with the parameters $h=0.2, \Delta t=0.01$ in the interval $-50 \leq x \leq 100$.

\begin{tabular}{lccc}
\hline \multicolumn{4}{c}{ Present method } \\
\hline$t$ & $I_{1}$ & $I_{2}$ & $I_{3}$ \\
\hline 0 & 40.50920 & 45.83608 & -37.87770 \\
10 & 40.45628 & 45.83603 & -34.73750 \\
20 & 40.46943 & 45.83597 & -34.97900 \\
30 & 40.55136 & 45.83593 & -33.83404 \\
40 & 40.45048 & 45.83586 & -33.69678 \\
50 & 40.34304 & 45.83577 & -33.17479 \\
$55[4]$ & 4040483 & 45.83524 & -32.37120 \\
$50[5](G A)$ & 40.41284 & 45.84364 & -32.14082 \\
$50[5](M Q)$ & 40.48389 & 45.85093 & -32.15991 \\
\hline
\end{tabular}

the interaction of two solitary waves. Thus, this numerical algorithm can be used reliably to obtain numerical solutions of the differential equations.

\section{REFERENCES}

1. Kawahara, T. 1972. Oscillatory waves in dispersive media. J. Phys. Soc. Japan 33, 260-264.

2. Boyd, J. P. 1991. Weakly non-local solitons for capillary-gravity waves: Fifth degree KdV equation. Physica D 48, 129-146.

3. Korkmaz, A. Dag, I. 2009. Crank-Nicolson-Differential quadrature algorithms for the Kawahara equation. Chaos Solitons Fractals 42, 65-73.

4. Haq, S. Uddin, M. 2008. RBFs approximation method for Kawahara equation. Engineering Analysis with Boundary Elements 35, 575-580.

5. Bibi, N. Tirmizi, S. I. A. Haq, S. 2011. Mesless method of lines for numerical solution of Kawahara type equations. Applied Mathematics 2, 608-618.

6. Yuan, J. M. Shen, J. Jiahong, W. 2008. A dual Petrov Galerkin method for the Kawahara-Type equations. Journal of Scientific Computing 34, 48-63.

7. Kaya, D. An explicit and numerical solutions of some fifth-order $\mathrm{KdV}$ equation by decomposition method. Applied Mathematics and Computation 144, 353-363.

8. Yusufoğlu, E. Bekir, A. 2008. Symbolic computation and new families of exact travelling solutions for the Kawahara and modified Kawahara equations. Computers and Mathematics with Applications 55, 1113-1121.

9. Jin, L. 2009. Application of Variational iteration method and Homotopy perturbation method to the modified Kawahara equation. Mathematical and Computer modelling 49, 573-578.

10. Yusufoglu, E. Bekir A. and Alp, M. 2008. Periodic and solitary wave solutions of Kawahara and modified Kawahara equations by Sine-Cosine method. Chaos,Solitons and Fractals 37, 1193-1197.

11. Prenter, P. M. 1975. Splines and Variational Methods, J. Wiley, New York.

12. Malik, R. P. 1997. On fifth orderKdV-type equation, Bogoliubov laboratory of theoretical physics. Joint Institute for Nuclear Research, Dubna, Moscow.

13. Yamamato, Y. Takizawa, E. 1981. On a solution on nonlinear time-evolution equation of fifth order. J. Phys. Soc. Jpn 50, 1421-1422.

14. Chang, Q. Wang, G. Guo, B. 1995. Conservative scheme for a model of nonlinear dispersive waves and its solitary waves induced by boundary motion. J. Comput. Phys. 93, 360-375. 